Tissue perfusion is the ultimate goal of a functional cardiocirculatory system and its integrity should remain the essential endeavor of all caregivers in charge of hemodynamically compromised patients. The postoperative cardiac patients are not the exception. Optimization of tissue perfusion after cardiac surgery is multifactorial and should be based upon invasive and non-invasive markers. Physiological elements to be considered and supported with medical therapy include: pre and afterload, cardiac contractility, heart rate and rhythm (determining cardiac output), analysis of systemic and pulmonary vascular resistances, evaluation of the systolic but also the diastolic function, and optimization of oxygen content, transport, extraction and consumption. All of the latter are to be managed taking into account elements related to microcirculation, cardiopulmonary and interventricular interactions and the neuro-humoral mechanisms triggered by the shock status. This conference will review decision-making processes, and therapeutic strategies related to general management principles, drugs (inotropic, lusitropic, vasodilator and vasoconstrictor medications), ventilation, and salvage therapies, amongst others.

\section{ADRENAL DYSFUNCTION IN CHILDREN REQUIRING CATECHOLAMINES AFTER CARDIAC SURGERY}

doi:10.1136/archdischild-2012-302724.0085

${ }^{1} \mathrm{R} G$ Branco, ${ }^{2} \mathrm{~A}$ Desai, ${ }^{1} \mathrm{~L}$ Filby, ${ }^{2} \mathrm{~L}$ Menadue, ${ }^{2} \mathrm{D} J \mathrm{Macrae} .{ }^{1}$ Paediatric Intensive Care Unit, Addenbrookes Hospital, Cambridge; 2 Paediatric Intensive Care Unit, Royal Brompton Hospital, London, UK

Background Adrenal dysfunction is associated with increased inotrope requirement and worse clinical outcome in children with sepsis. In children after bypass surgery a very low incidence of adrenal dysfunction is reported. However, use of hydrocortisone has been shown to improve hemodynamics of children requiring catecholamine after cardiac surgery. We aimed to evaluate the adrenal function of this subgroup of children requiring catecholamines after cardiac surgery.

Methods Retrospective analysis of adrenal function testing of children requiring catecholamine after cardiac surgery at the Royal Brompton Hospital from 2006 to 2011. Adrenal function was assessed using a $250 \mu \mathrm{g} / 1.73 \mathrm{~m}^{2} \mathrm{ACTH}$ stimulation test. Two definitions of adrenal dysfunction were used: physiological (peak cortisol after ACTH stimulation $<500 \mathrm{nmol} / \mathrm{L}$ ) and critical care (difference between cortisol post-stimulation and baseline cortisol $<250 \mathrm{nmol} / \mathrm{L}$ ). Children that received corticosteroids or etomidate, and children with known endocrine dysfunction were excluded.

Results 62 children were included in the study. Median (interquartile range) age was $3.2(0.9-6.5)$ months and weight $3.9(3.3-7.2) \mathrm{Kg}$. The mean RACHS was $2.5 \pm 0.7$ and median catecholamine score $0.11(0.06-0.18)$. Seven $(11.3 \%)$ children had adrenal dysfunction according to the physiological definition and 9 (14.5\%) according to the critical care definition. Children with physiological adrenal dysfunction had significantly higher catecholamine requirement than children with normal adrenal function $(p<0.01)$. There was no difference in catecholamine requirement in children with normal or abnormal adrenal function according to critical care definition.

Conclusions In children requiring catecholamines after cardiac surgery, adrenal dysfunction according to a physiological definition (but not critical care definition) is associated with higher catecholamine requirement.

\section{LOW-DOSE VASOPRESSIN IS CARDIOPROTECTIVE IN AN ACUTE SWINE MODEL OF NEONATAL HYPOXIA- REOXYGENATION}

doi:10.1136/archdischild-2012-302724.0086
'JS Pelletier, 'J LaBossiere, 'RS Gill, ${ }^{2} \mathrm{CM}$ Sergi, 'D Bigam, 'B Dicken, 1,3PY Cheung. 'Surgery; 'Laboratory Medicine and Pathology; ${ }^{3}$ Pediatrics, University of Alberta, Edmonton, $A B$, Canada

Background Cardiovascular dysfunction, a consequence of perinatal asphyxia, contributes significantly to its morbidity and mortality. The use of vasopressin, an endogenous hormone commonly given to adults with refractory shock, in neonates is limited by concerns over mesenteric perfusion. We recently showed that vasopressin had dose-dependent baro-specific effects with possible cardioprotection at low doses $(0.005-0.01$ units $/ \mathrm{kg} / \mathrm{h})$ in a swine model of neonatal hypoxia-reoxygenation (H-R). We aimed to compare systemic and regional hemodynamic effects of low-dose vasopressin and dobutamine, a synthetic beta-adrenoceptor agonist.

Methods Piglets (1-5 day-old, 1.6-2.2kg) were anesthetized and instrumented to continuously monitor systemic hemodynamic parameters including cardiac output (CO), and carotid and mesenteric flow indices. After $2 \mathrm{~h}$ of hypoxia $\left(10-15 \% \mathrm{O}_{2}\right)$, piglets had normoxic reoxygenation for $4 \mathrm{~h}$. In a blinded randomized fashion, piglets received either vasopressin $(0.01 \mathrm{units} / \mathrm{kg} / \mathrm{h}$ started at $30 \mathrm{~min}$ of reoxygenation) or dobutamine $(20 \mathrm{mcg} / \mathrm{kg} / \mathrm{min}$ started at $2 \mathrm{~h}$ of reoxygenation) ( $\mathrm{n}=8$ /group). H-R controls (placebo) and sham-operated piglets were also performed. Plasma troponin-I levels, tissue lactate levels and histology of left ventricle and small bowel were analyzed.

Results H-R piglets had cardiogenic shock and metabolic acidosis, which recovered upon reoxygenation. During recovery $\mathrm{CO}$, carotid and mesenteric flows gradually deteriorated and were increased similarly in vasopressin- and dobutamine-treated piglets ( $p<0.05$ vs. controls). Troponin, ventricular and intestinal lactate levels were reduced in vasopressin-treated piglets ( $p<0.05$ vs. controls), with no difference in histological analysis among groups.

Conclusion Low-dose vasopressin improves systemic and regional hemodynamics similarly to dobutamine and confers a cardioprotective effect in a swine model of neonatal $\mathrm{H}-\mathrm{R}$.

\section{ADVERSE DRUG EVENTS IN A PEDIATRIC INTENSIVE CARE UNIT: A PROSPECTIVE SURVEY}

doi:10.1136/archdischild-2012-302724.0087

1,2DC Bourguignon da Silva, ${ }^{3} \mathrm{OR}$ Araujo, ${ }^{3} \mathrm{RG}$ Arduini, ${ }^{3} \mathrm{CFR}$ Alonso, ${ }^{4} \mathrm{AR}$ Ogawa Shibata, ${ }^{4}$ EJ Troster. 'PICU, Universidade de São Paulo; ${ }^{2}$ Oncologic PICU; ${ }^{3}$ Universidade Federal de São Paulo; ${ }^{4}$ Universidade de São Paulo, São Paulo, Brazil

Background and aims Little is known about drugs pharmacokinetics and pharmodynamics in children. Renal and hepatic functions are not mature yet. Many drugs are used "off-label". So, children are more susceptible to adverse drug events (ADE) than adults. As many drugs are used in pediatric intensive care units (PICU), we expect to find many $\mathrm{ADE}$ in this setting. Our aims are to describe ADE found in PICU and identify risk factors for their development.

Methods Six-month prospective cohort of all patients admitted to a single PICU. ADE were identified by active search and classified by Naranjo's algorithm. Risk factors were identified by multivariate analysis.

Results 240 pediatric admissions occurred and $110 \mathrm{ADE}$ were observed in 84 patients. Median age was 51 months. Only 39 of 240 patients didn't have chronic status. Principal ADE were hyponatremia, hyperglycemia, hypokalemia. Drugs involved in most $\mathrm{ADE}$ were antibiotics, diuretics, antiepileptic, sedatives and analgesics and steroids. Age under 4 years, length of stay in PICU and number of drugs used are risk factors to $\mathrm{ADE}$.

Conclusions There were $64.7 \mathrm{ADE} / 1000$ patients-day in the present study. Hyponatremia, hypokalemia and hyperglycemia are the 